\title{
EFFECT OF SLICE STEP SIZE ON PREDICTION OF NATURAL VIBRATION PROPERTIES OF BONE TISSUE
}

\author{
Gokhan ALTINTAS \\ Department of Mechanical Sciences, Engineering Faculty, Celal Bayar University, \\ Muradiye. 45100, Turkey
}

\begin{abstract}
Several vibration analysis procedures are used for determination of the level of bone loss, status of implant stability, modal damping factor and numerous other properties of tissues. The detection methods of bone properties are to compare the results of theoretical work with practical results. So, there are many options for processing of image data and establishing the finite element (FE) model that differentiation of calculated outputs is inevitable. Uncertainty of outputs can lead to mistakes while mechanical parameters or behaviors of tissue are determined. In this study, the effect of Micro-CT scanning intensity in connection with the reconstruction process on properties of the modal behavior of bone tissue were investigated. Results have shown that examined parameters have important effects on numerical values of the natural frequencies and modal behaviors. Furthermore, it has been revealed that numerical values and mode shapes must be considered together for properly understanding the natural vibration analysis of bone tissue.
\end{abstract}

Key Words-Slice Step Size, Bone Tissue, Slice Thickness, Modal Analysis, Voxel Size

\section{INTRODUCTION}

Not only the usage of the detection methods with vibration but also vibration properties of bones is important for bone diseases. Investigations published by Cornelissen et al. $[1,2]$ and Van der Perre and Lowet [3, 4] mainly studied the determination of free vibration frequencies and corresponding mode shapes of human bones, particularly the tibia and the radius. The results are shows that the natural frequencies of bone decrease with increasing degree of osteoporosis. Lowet et al. [5] concluded that bending modes and stiffness of long bones are directly accompanying with its natural frequencies. The verification of a non-invasive mechanical measurement of bone by comparison to actual values was determined by Robertsa et al. [6].

Determination of mechanical properties of bone tissue has a key role in diagnosis and treatment of bone diseases. The role of natural frequency of bone for detection of bone fracture healing was investigated by Singh et al. [7]. Nokes et al. [8] studied by using direct and indirect methods for natural frequency of tibial. Christopoulou et al. [9] aimed to understand the potential of modal damping factor as a diagnostic tool for osteoporosis and to study the effects of alendronate on damping factor and the bone biochemical markers NTx and osteocalcin. Kotha et al. [10] examined if high frequency ultrasound impedance measurements could forecast the mechanical properties of bones where the amount of bone minerals was varied. Mc Donnell et al. [11] practices rapid prototype replicas produced based on Micro-CT data for disclosing variations in the mechanical properties due to progressive bone loss. Bediz et al. [12] set vibration 
properties, mainly the loss factor of the tibia acquired from frequency response function gets by vibration analysis both in vivo and in vitro tests.

Through technological improvements, high-resolution scans can be acquired by MicroCT or Micro-MRI techniques in the order of a few microns per pixel. Spatial resolution is the quality of an image, or its ability to distinguish tiny objects and to determine clearly edges and gaps. Micro-architectural structures considered in the analysis, and realistic models can be acquired by high-resolution scanning data. Ulrich et al. [13] utilised a scanner to analysis the relationship between micro-architectural properties Parfitt [14] and mechanical parameters of cancellous bone. Scanned image sets of cancellous bone samples at a micron resolution were acquired from these datasets. They set micro-architectural properties as well as converted them into Micro Finite Element $(\mu \mathrm{FE})$ models. These $\mu \mathrm{FE}$ models were used to compute orthotropic mechanical stiffness; usage of $\mu \mathrm{FE}$ models were to ensure that stiffness constants evaluated was purely due to the Micro-Architecture, and not to trabecular bone tissue properties and experimental artifacts.

Chevalier et al. [15] examined that, voxel-based FE analysis of trabecular bone is combined with physical measures of the volume fractions, Micro-CT reconstructions, uniaxial mechanical tests and nano-indentation tests for appropriate validation of the method. To this end, micro-imaging techniques and $\mu \mathrm{FE}$ can be used to evaluate the load transfer within the developed scaffolds [16,17]. This method permits the reconstruction of small samples [16]. The reconstructed geometry can be used for the evolution of $\mu \mathrm{FE}$ models to simulate load transfer in bone remodeling [18] and can be merged with scanning techniques to be obtained fully automated reconstructed samples [19].

Influence of voxel size and segmentation methods on FE models analysed several researchers. FE calculations of apparent modulus were already expressed to be strongly influenced by the threshold used for segmentation of CT data to acquire the FE mesh Hara et al. [20], and are highly sensitive to errors due to the strong relationship between volume fraction and mechanical properties.

Bevill and Keaveny [21] they sought to settle whether forecasting of yield stress provided by nonlinear FE models could improve correlations with bone strength as compared to the application of anticipations of young modulus from linear FE models, and if this effect depended on a voxel size or bone volume fraction.

Literature survey shows that there is no study addressed the effect of slice step size (shortly step size or slice thickness) on natural vibration properties of bone tissue. This study set out with the aim of assessing the importance of slice thickness in natural vibration properties and behaviors of trabecular bone tissue in micro scale. In addition, we examine the effect of slice thickness on model geometry and FE mesh naturally.

\section{MATERIAL AND METHODS}

The Micro-CT scanning images (TIFF files) are processed by a 2D segmentation script in MATLAB ${ }^{\circledR}$. The data set contains a images of human lumbar vertebral body L3. The original data is stored as 900 slices, each of size $1012 \times 1012$. In this work region of interest (ROI) contains 121 slices and $100 \times 100$ pixel size in the case of isotropic voxel. The grayscale pixel values of the images were linearly scaled to cover the range from 0 
to 255 having minimum and maximum values, respectively. Threshold range from 136 to 255 of was used.
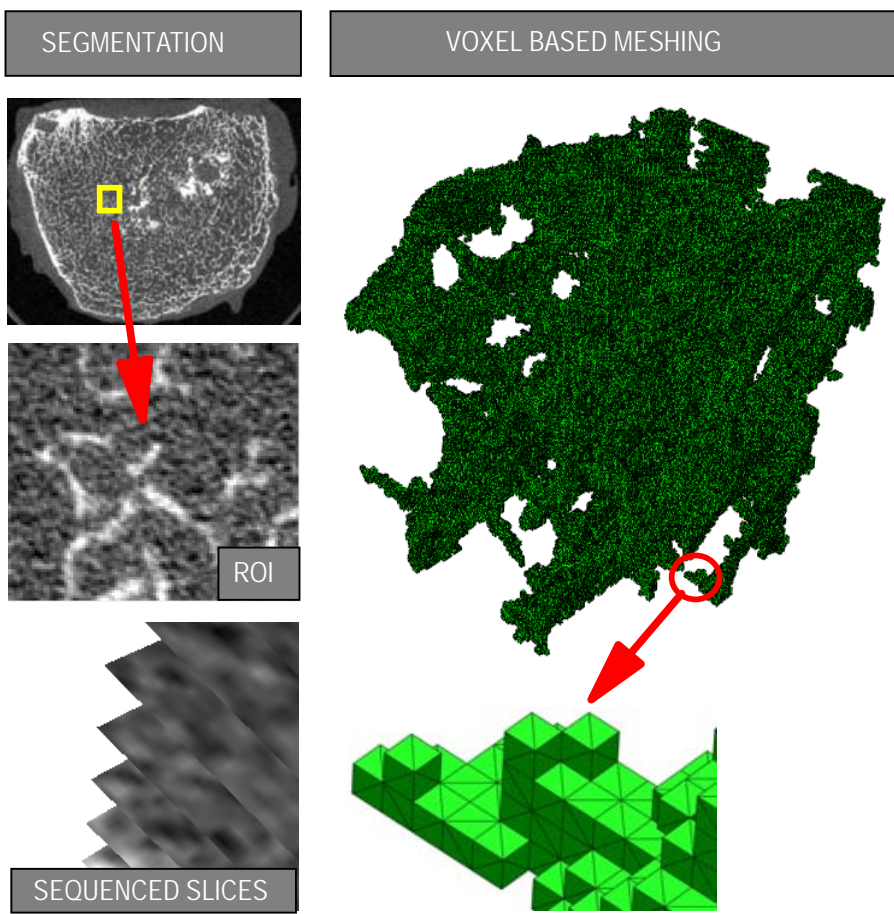

RESULTS OF FINITEELEMENT MODEL

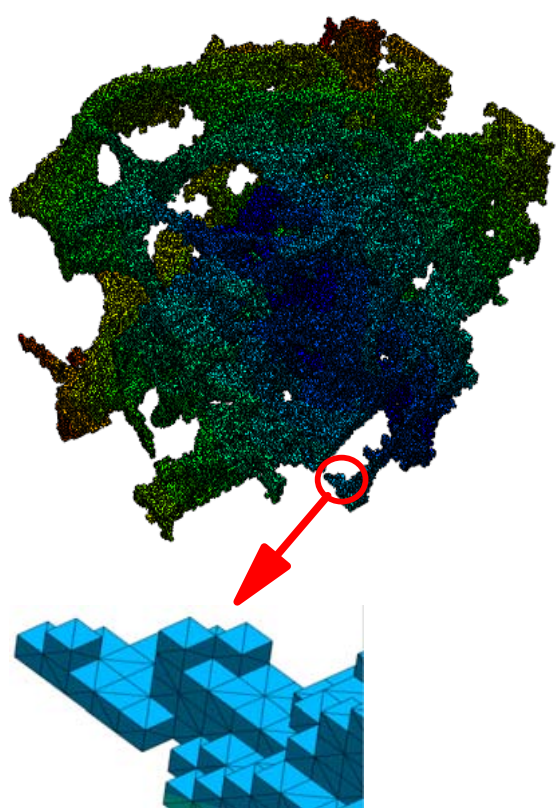

Figure 1. Methodology for 2D images to FEM and FEA Results

As the pixel width and pixel length are known, each pixel can be assumed to shape a basis of a brick with the height equal to the slice thickness. $\mu \mathrm{CT}$ image files $(37-\mu \mathrm{m}$ isotropic voxel size) were first binarized at the midpoint between the peaks of bone intensities of the histogram. Anisotropic voxels with high 74, 111, and $148 \mu \mathrm{m}$ were also reconstucted while keeping the base area $37 \times 37 \mu \mathrm{m}^{2}$. Each voxel is split into five tetrahedra. To ensure that connection of cells match each other, the orientations of the tetrahedra changed by Cutler [22]. In tomographic images discrete voxels are utilized to describe continuum bone tissue. Even so, discrete voxels derived by threshold do not guarantee continuum bone tissue. 3D Flood fill script was written in Matlab ${ }^{\circledR}$ for providing interconnected bone tissue model. Additionally Flood fill algorithm removes islands from the bone tissue model. Islands can arise from noisy images or threshold errors and cause analysis failures. Segmentation and reconstruction procedures are used in its simplest form for focusing only effect of slice thickness additionally secondary algorithms like surface smoothing and others are not used in analyses. In some image processing stages, we frequently used software developed by the NIH/NCRR Center for Integrative Biomedical Computing.

Modal analysis was performed by using the commercial solver ABAQUS ${ }^{\circledR}$ with the material properties (Young modulus 17.3 GPa, Possion Ratio 0.3, Density $1874 \mathrm{~kg} / \mathrm{m}^{3}$ ) and free boundary conditions. 


\section{RESULTS AND DISCUSSION}
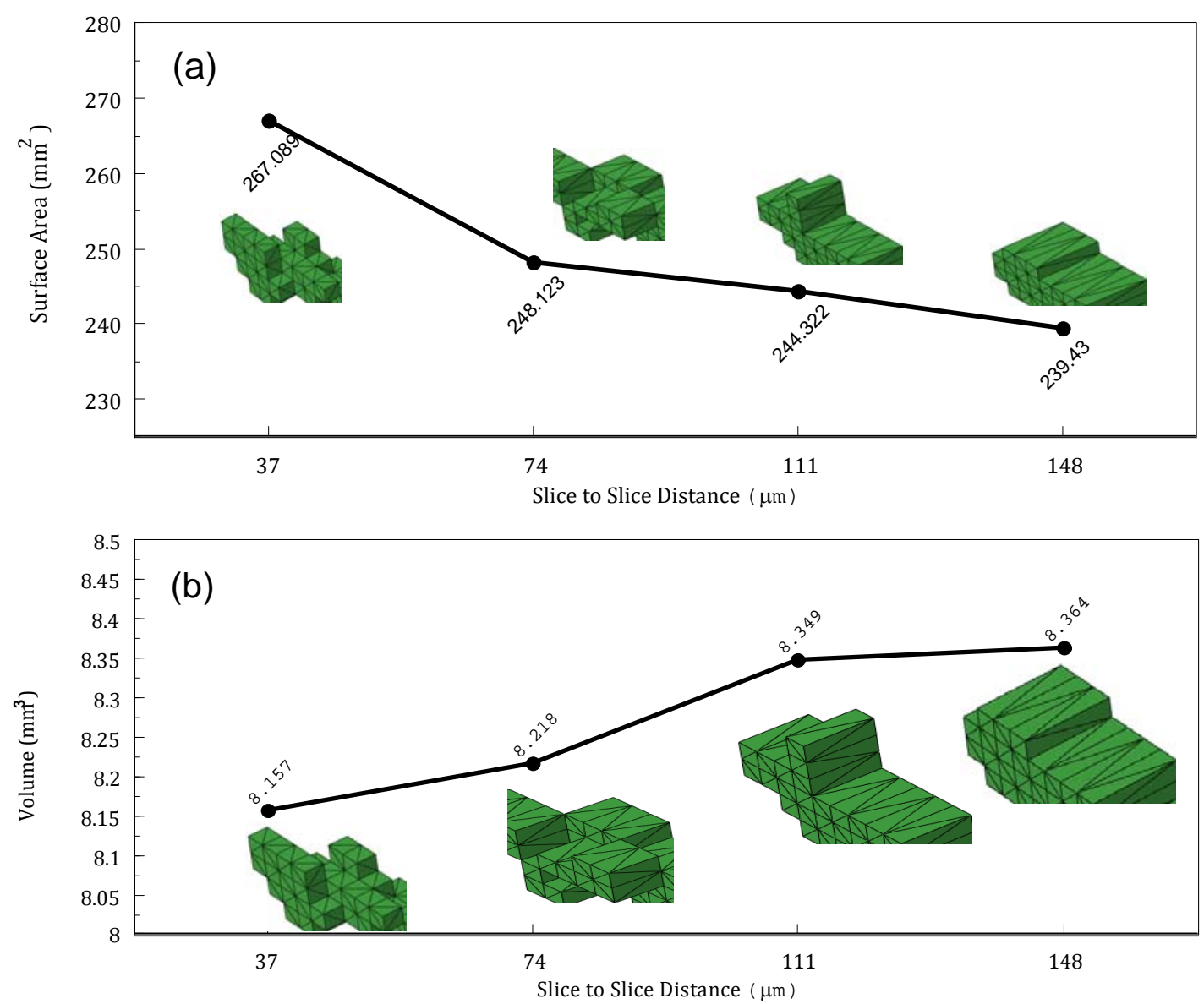

Figure 2. Effect of Slice Step Size on Geometric Properties: (a) Area, (b) Volume

Primary geometric properties of 3D solid models are shown in Fig. 2 for four different slice step. Surface area of the models decrease while slice step increases. On the other hand, the volume of the models increased for decreasing of slice step.

A difference in surface area between models with $37 \mu \mathrm{m}$ and $148 \mu \mathrm{m}$ slice thickness reaches $\% 10.3$ while difference in volume is $\% 2.5$ for the same slice thickness values. Apparently, slice thickness is more effective on surface area than volume of models. This issue can be important for analysis like fluid flow and tissue interaction problems where surface area more dominant than model volume in many cases. Examined geometrical properties of generated models were shown that the effect of slice step on surface area, and volume is unpredictable and irregular.

The area and volume of bone structure do not change linearly for constant increment of slice thickness contrary to prismatic shapes with constant cross section. To distinguish bone tissue in Micro-CT images threshold technique was used. Flood fill algorithm applied for removing islands and providing interconnections of bone tissue. 

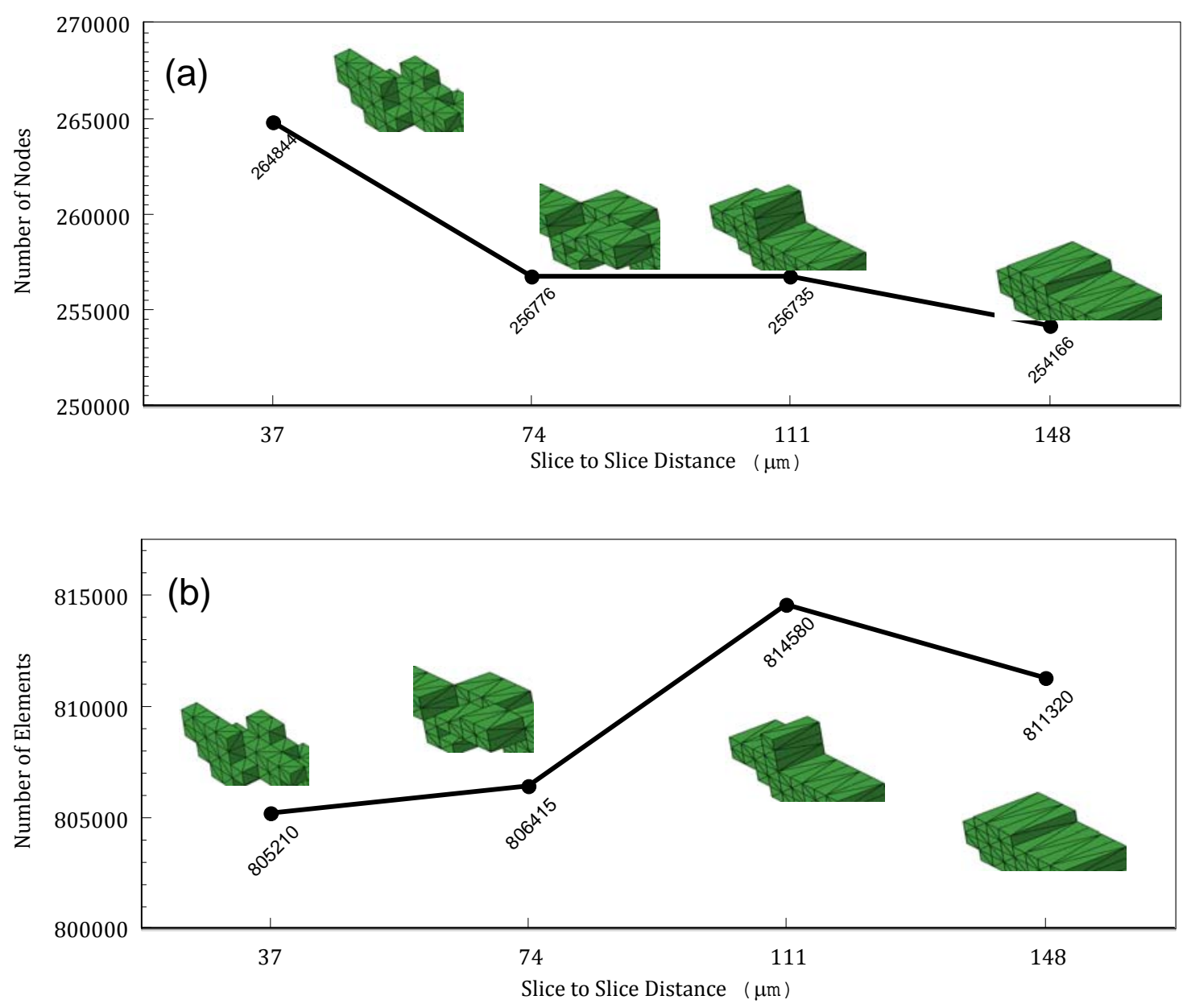

Figure 3. Effect of Slice Step Size on Mesh Properties: (a) Number of Nodes, (b) Number of Elements

Unlike structured meshed prismatic systems with simple projections, it is not observed that relationship with a constant trend between the number of nodes and elements. For the range of the inspection interval, number of nodes of generated meshes inversely related to slice thickness while the irregular relationship exists between slice thickness and number of elements.

Table 1. Natural Frequencies for Voxel Size.

\begin{tabular}{|c|c|c|c|c|c|c|c|c|c|c|c|}
\hline & \multicolumn{10}{|c|}{ Mode Number } \\
\hline & & 1 & 2 & 3 & 4 & 5 & 6 & 7 & 8 & 9 & 10 \\
\hline \multirow{8}{*}{$\begin{array}{l}\stackrel{\tilde{N}}{\tilde{n}} \\
\overrightarrow{0} \\
\ddot{x} \\
\dot{\nu}\end{array}$} & $37 \times 37 \times 37$ & 3014 & 3302 & 3342 & 4214 & 5137 & 5729 & 5883 & 5971 & 6274 & 6622 \\
\hline & & 7 & 8 & 3 & 6 & 2 & 7 & 2 & 0 & 2 & 4 \\
\hline & $37 \times 37 \times 74$ & 3180 & 3240 & 3525 & 4099 & 4871 & 5517 & 5735 & 5851 & 6260 & 6572 \\
\hline & & 2 & 8 & 6 & 7 & 0 & 9 & 9 & 1 & 8 & 6 \\
\hline & $37 \times 37 \times 11$ & 3006 & 3145 & 3710 & 4043 & 4529 & 5214 & 5383 & 5691 & 5944 & 6353 \\
\hline & 1 & 7 & 8 & 4 & 4 & 5 & 2 & 9 & 2 & 7 & 8 \\
\hline & $37 \times 37 \times 14$ & 2410 & 2856 & 3312 & 3538 & 3643 & 4002 & 4076 & 4187 & 4574 & 4824 \\
\hline & 8 & 9 & 5 & 5 & 1 & 4 & 1 & 1 & 0 & 5 & 5 \\
\hline
\end{tabular}


Table 1 shows the first ten natural frequency of systems with different slice thickness. For increasing slice thickness modes occurred in a narrowing range of frequency. Shortly no clear relationship between natural frequencies of models with different mesh, therefore, the more detailed table below created to see modal behavior of modes by observing mode shapes and frequencies together.

Table 2. Natural Frequencies and Associated Mod Shapes for Mesh Types.

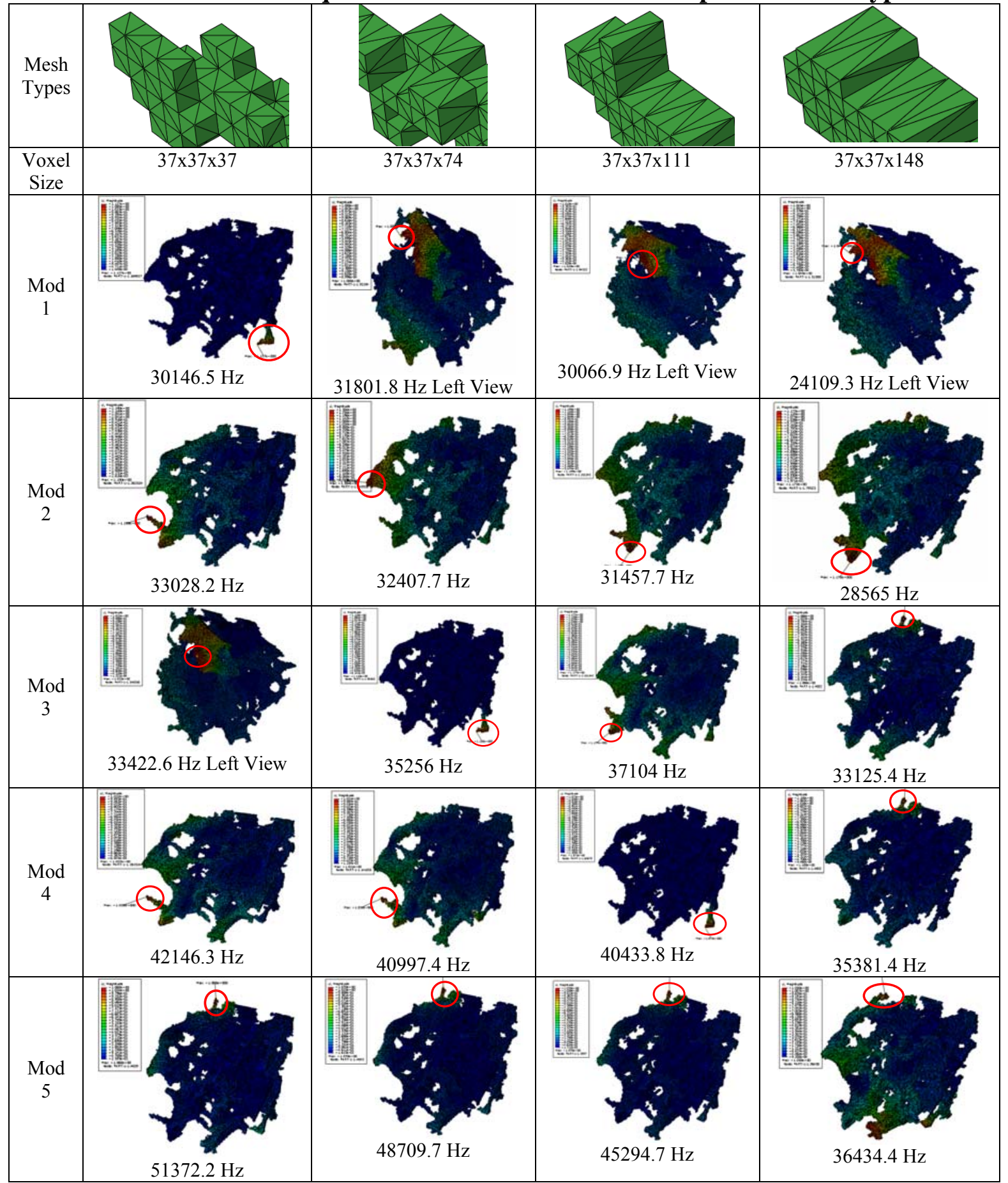


In Table 2, natural frequencies and related mod shapes with displacement contour are shown together. Maximum displacement area of each mod shape of the bone model marked with ring.

The maximum displacement region of each mode shape is a quite small region for displayed modes in Table 2. Beyond the maximum displacement region displacements are an almost constant amount, and the region acts like a support. This behavior can be an important consideration in the case of osteoclasis and micro applications with vibration.

It can be seen from the Table 2, there is no clear relationship between mode types and mode sequences for each model. Each model has its own mode sequence, and number of frequencies are different also for same mode types. One of the important results obtained from this study is that the natural frequency values and mod shapes must be considered together for free vibration behavior of models that derived from sets with different slice thickness is surprisingly different from each other. These differences include not only natural frequencies but also mode types and sequences.

Another effect of modal behavior irregularity is vibration behaviors of models unpredictable by using results of other models with different slice thickness values.

Although variation of slice thickness effects to the element size the main changes occurred in model geometry. Effects of segmentation methods on geometric identities are crucial when the amount of missing details is increased due to usage of large slice thickness. This issue is especially important in the case of fatal bone loss when weak connections come out. Element size effect on analysis types in finite element techniques is well known in established literature but this interesting result cannot be explained by only the variation of element size.

The changes of element size and model geometry together combined effect cause irregular modal behavior, contrary to the case of element size variation in routine FE techniques. Generally, in the usage of FE method, the variation of element sizes affects the only convergence level, but it does not change trends generally. The variation of slice thickness accompanied by segmentation techniques can cause not only the change of element size but also ignorance of weakly connected parts or production of parts, which is not existing in real. The combined effects of variation of slice thickness and segmentation method caused irregular trends and convergence levels in vivo. At last, the results revealed that $3 \mathrm{D}$ solid FE models must be reconstructed by using Micro-CT images with using smallest slice thickness.

\section{CONCLUSIONS}

This assignment was explained the central importance of slice thickness in free vibration properties of cancellous bone. The most obvious finding emerging from this study is that the natural vibration behavior of models with different slice thickness is surprisingly different from each other in terms of not only the values of natural frequencies but also modal behavior.

One of the more significant findings of this study is that changes of element size and model geometry together causes inconsistent modal behavior. The findings of this study have a number of important implications for in the process of reconstruction and analysis in future practice. 
The current study was specifically designed to evaluate the influence of slice thickness on free vibration properties related to anisotropic voxel size. Therefore, the findings might not be transferable to virtual models derived using different solid model evaluation processes. This research has thrown up many questions in need of further investigation. Taken together, these results suggest that 3D model reconstruction must be done shortest slice thickness and model behavior consideration must be included both natural frequency values and mode shapes.

\section{REFERENCES}

1. M. Cornelissen, P. Cornelissen, G. van der Perre, A.B. Christensen, F. Ammitzboll, C. Dyrbye, Assessment Of Tibial Stiffness By Vibration Testing In Situ - III. Identification Of Mode Shapes In Different Supporting Conditions, Journal of Biomechanics, 20, 333-342, 1986.

2. M. Cornelissen, P. Cornelissen, G. van der Perre, A.B. Christensen, F. Ammitzboll, C. Dyrbye, Assessment Of Tibial Stiffness By Vibration Testing In Situ - II. Influence Of Soft Tissues, Joints And Fibula, Journal of Biomechanics, 19, 551-561, 1986.

3. G. van der Perre, G. Lowet, Vibration, Sonic And Ultrasonic Wave Propagation Analysis For The Detection Of Osteoporosis, Clinical Rheumatology, 13, 45-53, 1994.

4. G. van der Perre, G. Lowet, In-Vivo Assessment Of Bone Mechanical Properties By Vibration And Ultrasonic Wave Propagation Analysis, Bone, 18, 29- 35, 1996.

5. G. Lowet, R. van Audekercke, G. van der Perre, P. Geusens, J. Dequeker, J. Lammens, The Relation Between Resonant Frequencies And Torsional Stiffness Of Long Bones In Vitro Validation Of A Simple Beam Model, Journal of Biomechanics, 26, 689-696, 1993.

6. S. G. Roberts, T. M. Hutchinson, S. B. Arnaud, B. J. Kiratli, R. B. Martin, C. R. Steel, Noninvasive Determination Of Bone Mechanical Properties Using Vibration Response: A Refined Model And Validation In Vivo, Journal of Biomechanics, 29, 9198, 1999.

7. V.R. Singh, S. Yadav, V.P. Adya, Role Of Natural Frequency Of Bone As A Guide For Detection Of Bone Fracture Healing, Journal of Biomechanical Engineering, 11, 457-461, 1989.

8. L. Nokes, W.J. Mintowt, I. Mackie, J.A. Fairclough, J. Williams, Direct and indirect determination of tibial natural frequency - A comparison of frequency domain analysis and Fast Fourier Transform, Journal of Biomedical Engineering, 6, 45-48,1984.

9. G.E. Christopoulou, A. Stavropoulou, G. Anastassopoulos, S.D. Panteliou, E. Papadaki, N.K. Karamanos, E. Panagiotopoulos, Evaluation Of Modal Damping Factor As A Diagnostic Tool For Osteoporosis And Its Relation With Serum Osteocalcin And Collagen I N-Telopeptide For Monitoring The Efficacy Of Alendronate In Ovariectomized, Journal of Pharmaceutical and Biomedical Analysis, 41, 891-897, 2006.

10. S. P. Kotha, C. A. De Paula, A. B. Mann, N. Guzelsu, High Frequency Ultrasound Prediction of Mechanical Properties of Cortical Bone with Varying Amount of Mineral Content, Ultrasound in Medicine and Biology, 34, 630-637, 2007. 
11. P. Mc Donnell, M.A.K. Liebschner, W. Tawackoli, P.E. Mc Hugh, Vibrational testing of trabecular bone architectures using rapid prototype models, Medical Engineering \& Physics, 31, 108-115, 2009.

12. B. Bediz, H. N. Özgüven, F. Korkusuz, Vibration Measurements Predict The Mechanical Properties Of Human Tibia, Clinical Biomechanics, 25, 365-371, 2010.

13. D. Ulrich, B. Van Rietbergen, A. Laib, P. Ruegsegger, The Ability Of ThreeDimensional Structural Indices To Reflect Mechanical Aspects Of Trabecular Bone, Bone, 25, 55-60, 1999.

14. A.M. Parfitt, Bone Histomorphometry: Standardization Of Nomenclature, Symbols And Units, Bone, 9, 67-69, 1988.

15. Y. Chevalier, D. Pahr, H. Allmer, M. Charlebois, P. Zysset, Validation Of A VoxelBased FE Method For Prediction Of The Uniaxial Apparent Modulus Of Human Trabecular Bone Using Macroscopic Mechanical Tests And Nanoindentation, Journal of Biomechanics, 40, 3333-3340, 2007.

16. W. Sun, B. Starly, J. Nam, A. Darling, Bio-CAD Modeling And Its Applications In Computer-Aided Tissue Engineering, Computer-Aided Design, 37, 1097-1114, 2005.

17. S.V.N. Jaecques, H. Van Oosterwyck, L. Muraru, T. Van Cleynenbreugel, E. De Smet, M. Wevers, Individualised, Micro CT-Based Finite Element Modelling As A Tool For Biomechanical Analysis Related To Tissue Engineering Of Bone, Biomaterials, 25, 1683-96, 2004.

18. R.B.G. Breuls, B.G. Sengers, C.W.J. Oomens, C.V.C. Bouten, F.P.T. Baaijens. Predicting Local Cell Deformations In Engineered Tissue Constructs: A Multilevel finite Element Approach, Journal of Biomechanical Engineering, 124, 198-207, 2002.

19. J.M. Williams, A. Adewunmi, R.M. Schek, C.L. Flanagan, P.H. Krebsbach, S.E. Feinberg, Bone Tissue Engineering Using Polycaprolactone Scaffolds Fabricated Via Selective Laser Sintering, Biomaterials, 26, 4817-4827, 2005.

20. T. Hara, E. Tanck, J. Homminga, R. Huiskes, The Influence Of Microcomputed Tomography Threshold Variations On The Assessment Of Structural And Mechanical Trabecular Bone Properties, Bone, 31, 107-109, 2002.

21. G. Bevill, T. M. Keaven, Trabecular Bone Strength Predictions Using finite Element Analysis Of Micro-Scale Images At Limited Spatial Resolution, Bone, 44, 579-584, 2009.

22. B. Cutler, J. Dorsey, L. Mcmillan, M. Müller, R. Jagnow, A Procedural Approach To Authoring Solid Models, ACM Transactions on Graphics, 302-311, 2002. 